\title{
Biological intervertebral disc replacement: an in vivo model and comparison of two surgical techniques to approach the rat caudal disc
}

Authors

Institutions
Harry Gebhard ${ }^{1,2 *}$, Andrew R James ${ }^{1}$, Robby D Bowles ${ }^{3}$, Jonathan P Dyke ${ }^{4}$, Tatianna Saleh ${ }^{1}$, Stephen P Doty ${ }^{5}$, Lawrence J Bonassar ${ }^{6}$, and Roger Härtl ${ }^{1}$

${ }^{1}$ Brain and Spine Center, Department of Neurosurgery, Weill Cornell Medical College, New York, NY, USA

${ }^{2}$ Department of Trauma Surgery, Technical University, Munich, Germany

${ }^{3}$ Department of Biomedical Engineering, Cornell University, Ithaca, NY, USA

${ }^{4}$ Department of Radiology, Weill Cornell Medical College, New York, NY, USA

${ }^{5}$ Research Division, Hospital for Special Surgery, New York, NY, USA

${ }^{6}$ Department of Biomedical Engineering and Sibley School of Mechanical and Aerospace Engineering, Cornell University, Ithaca, NY, USA

\section{ABSTRACT}

Study design: Prospective randomized animal study.

Objective: To determine a surgical technique for reproducible and functional intervertebral disc replacement in an orthotopic animal model.

Methods: The caudal 3/4 intervertebral disc (IVD) of the rat tail was approached by two surgical techniques: blunt dissection, stripping and retracting (Technique 1) or incising and repairing (Technique 2) the dorsal longitudinal tendons. The intervertebral disc was dissected and removed, and then either discarded or reinserted. Outcome measures were perioperative complications, spontaneous tail movement, 7T MRI (T1- and T2-sequences for measurement of disc space height (DSH) and disc hydration). Microcomputed tomographic imaging (micro CT) was additionally performed postmortem.

Results: No vascular injuries occurred and no systemic or local infections were observed over the course of 1 month. Tail movements were maintained. With tendon retraction (Technique 1) gross loss of DSH occurred with both discectomy and reinsertion. Tendon division (Technique 2) maintained DSH with IVD reinsertion but not without. The DSH was demonstrated on MRI measurement. A new scoring system to assess IVD appearances was described.

Conclusions: The rat tail model, with a tendon dividing surgical technique, can function as an orthotopic animal model for IVD research. Mechanical stimulation is maintained by preserved tail movements. 7T MRI is a feasible modality for longitudinal monitoring for the rat caudal disc.

* The experiments were performed at Weill Cornell Medical College, New York, NY, USA.

Roger Härtl and Harry Gebhard received a grant from AOSpine North America for this study. 


\section{STUDY RATIONALE AND CONTEXT}

Recent work introduced tissue-engineered intervertebral disc (IVD) constructs, which have shown promising characteristics in vitro [1-4]. An orthotopic model is the desirable next step to provide biological and mechanical stimulation. We describe a new surgical approach and animal study, to our knowledge not previously reported, to demonstrate feasibility of an in vivo model and to compare the influence of surgical disc space dissection on survival of a reimplanted disc [5]. Using magnetic resonance imaging (MRI), sequential assessments are made and a new scoring system is described.

\section{CLINICAL QUESTIONS}

Does the surgical approach to the IVD in the rat tail, with discectomy or disc excision and reimplantation alter the postoperative disc height and appearance of the IVD on MRI? Is this a suitable animal model for preliminary IVD replacement testing using noninvasive means in longitudinal studies?

\section{METHODS}

Study design: Prospective randomized animal study.

- Local ethical and animal committee approval obtained.

- 24 nude (athymic) adult male rats, 250-300 g (Hsd: RH-Foxnlrnu) were included, the exclusion criterion was previous tail injury. No animals were excluded.

- Same surgeon for anesthesia (induction: $85 \mathrm{mg} / \mathrm{kg}$ Ketamine and $5 \mathrm{mg} / \mathrm{kg}$ Xylazine administered IP; maintenance: Isoflurane $2 \%$ ) and surgery.

- Intraoperative x-rays located the caudal 3/4 disc. The operative approach involved skin incisions and a midline muscle flap to be created identically in both groups (Fig 1). The paravertebral longitudinal tendons were then either:

- Retracted with blunt dissection and tissue stripping (Technique 1; Fig 2)

- Divided by sharp incision (Technique 2; Fig 3)

- Surgical sharp dissection was performed around the disc. Only then the annular fibers were sharply incised (cranial and caudal) and the disc was removed as a whole with blunt detachment of the nucleus (Fig 4).
- Next the disc was either discarded (discectomy) or reimplanted.

- Technique 2: Divided tendons underwent a tensionfree surgical repair.

- In both techniques, a saline wash was performed, the muscle was reinserted, and the skin was sutured. Postoperative analgesia (Buprenorphine for 72 hours) was administered and the animals were under observation until active and feeding.

\section{Outcomes}

- Clinical assessments:

- Daily wound observation (infection, delayed closure).

- Vascular assessment: Normal capillary blood flow at the rat tail tip was assessed clinically by digital pressure and the ability of the tip to reperfuse within 2 seconds.

- Function: At day 29 all animals were visually observed and tail movements counted by a singleskilled assessor during awake, day and night, at 30-minute periods. Internal control consisted of preoperative observations of six animals.

- MRI was performed day 1 and day 30.

- DSH was measured on Tl sequences (Fig 5) using an image analysis system (Osirix viewer ${ }^{\circledR}$ ).

- IVD characteristics were measured on the T2 sequences, using a semiquantitative scoring system as shown in Table 2 (Fig 6).

- Magnetic resonance angiography (MRA) was conducted day 30 to assess for vascular injury (Fig 7).

- Two-blinded observers assessed MRI scans. The DSH was averaged, disagreement on characterization or vascular injury were decided by an independent third person, whose grading would be final.

- Micro CT: High-resolution images postmortem to assess injuries to vertebrae and end plates (Fig 8).

Analysis: Distinct results were interpreted directly, while variations between groups were tested statistically.

Clinical assessment: Wound complications (infection, delayed closure), incidence of capillary delay, and loss of vessel integrity were counted and compared with a $t$ test.

- The tail-bending frequencies were analyzed with a Poisson regression model, and a multivariate analysis with stepwise variable selection between the groups.

- For disc height and disc grading, the adjacent level was the comparator. The strength of collapse measured by the relation of preoperative and postopera- 
tive surgical gap size was tested against a threshold of one, representing no change, by means of twosided one-sample $t$ tests. Comparisons of postoperative relations and corresponding values after 1 month were conducted by two-sided paired-samples $t$ tests. Likewise it was tested for differences between relations observed with and without disc reinsertion, and between surgical approaches by two-sided two-sample $t$ tests. In addition descriptive statistics are given by mean, standard deviation, and 95\% confidence intervals. All tests were performed in an explorative manner on a 5\% level of significance. Tabulation of scores and calculation of the Fleiss' kappa coefficient for reliability testing in multirater settings.

- Number of injuries to the bony end plates was recorded.

Fig 2 Diagram with tendon retraction and midline muscle flap created.

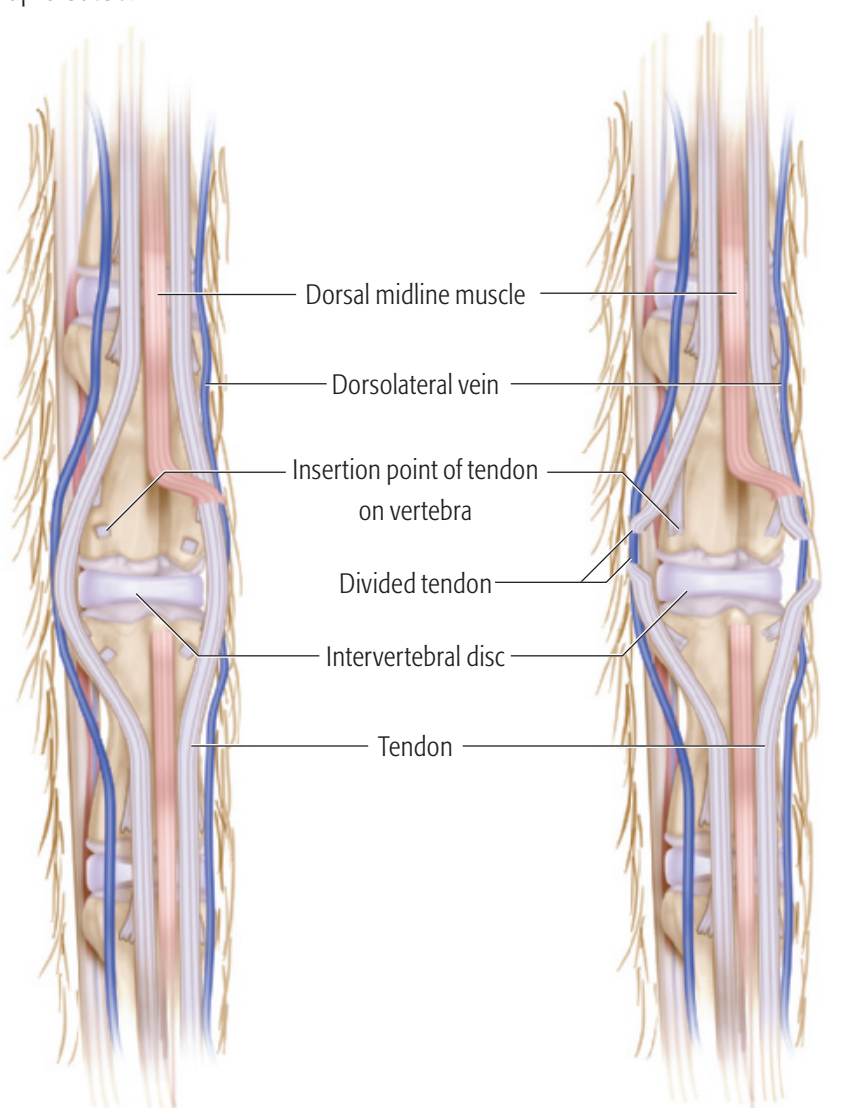

Fig 3 Diagram with incised tendons and midline muscle flap.
Fig 1 Intraoperative appearance of the longitudinal paravertebral tendons after skin flaps are created.

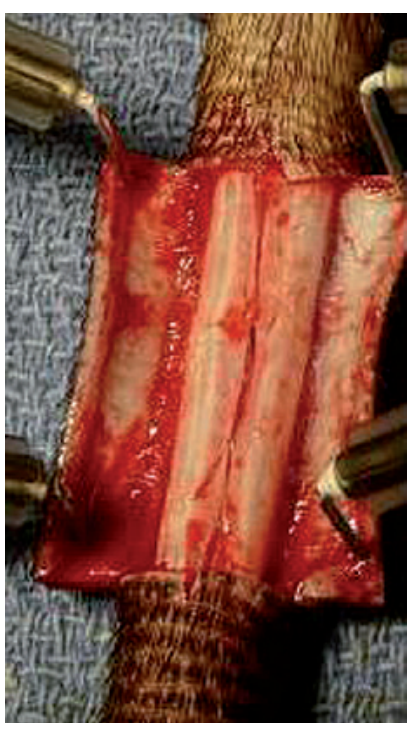

Fig 4 Explanted native disc.

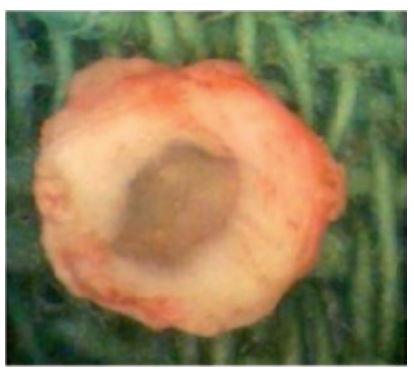

Fig 5 Magnetic resonance imaging. T1-weighted image showing disc height. Reimplanted disc is shown in center.

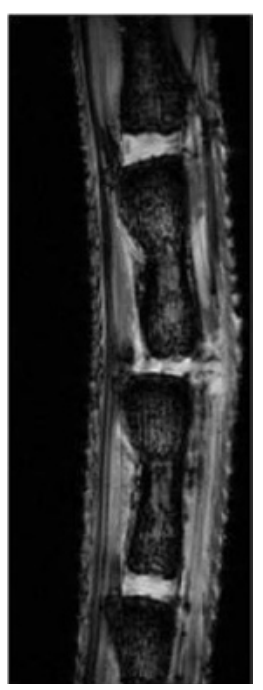


Fig 6 Magnetic resonance imaging (T2 sequences) showing hydration and characteristics of IVD/disc space as explained in Table 2.

Level of surgery shown in center (Grades 0-3). Grade 4 demonstrates a native level without surgery. Scoring examples are displayed.

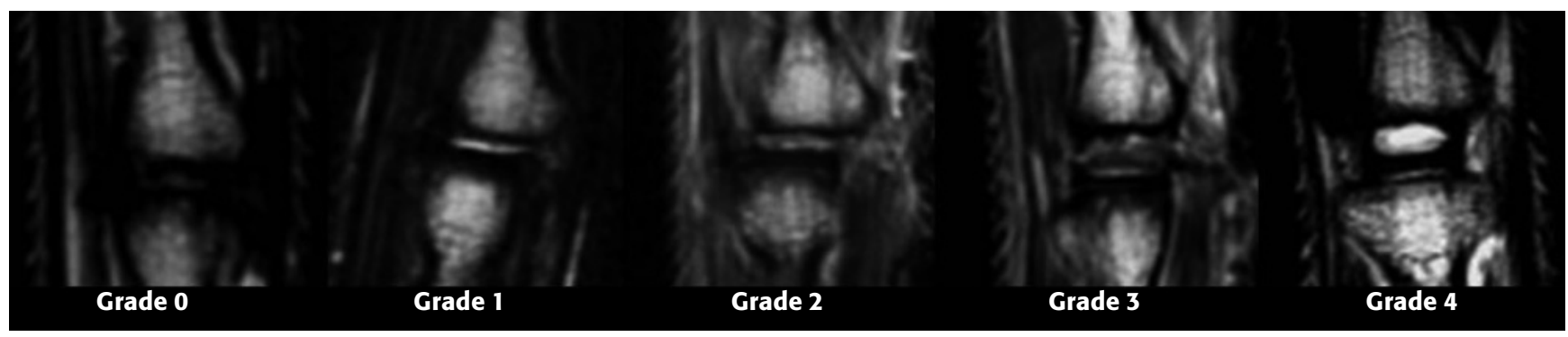

Fig 7 Magnetic resonance angiography of rat tail in sagittal midline imaging. The vasculature is intact.

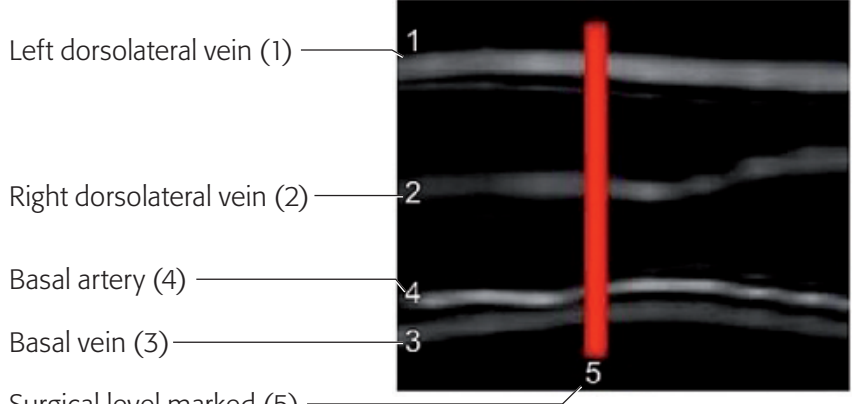

Surgical level marked (5) (vertical line)
Fig 8 Microcomputed tomographic scans showing sagittal midline image of adjacent levels, with axial images of the distal end plates at each level below. The disc has been excised on the left, and on the right is an intact adjacent level. There is no bony injury demonstrated.

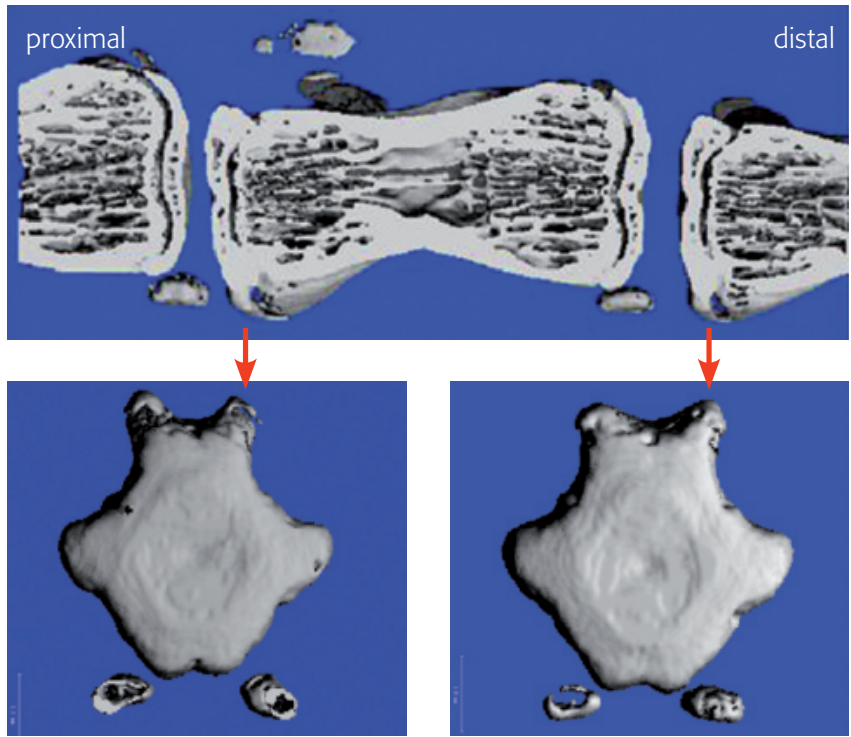

Fig 9 Disc height.

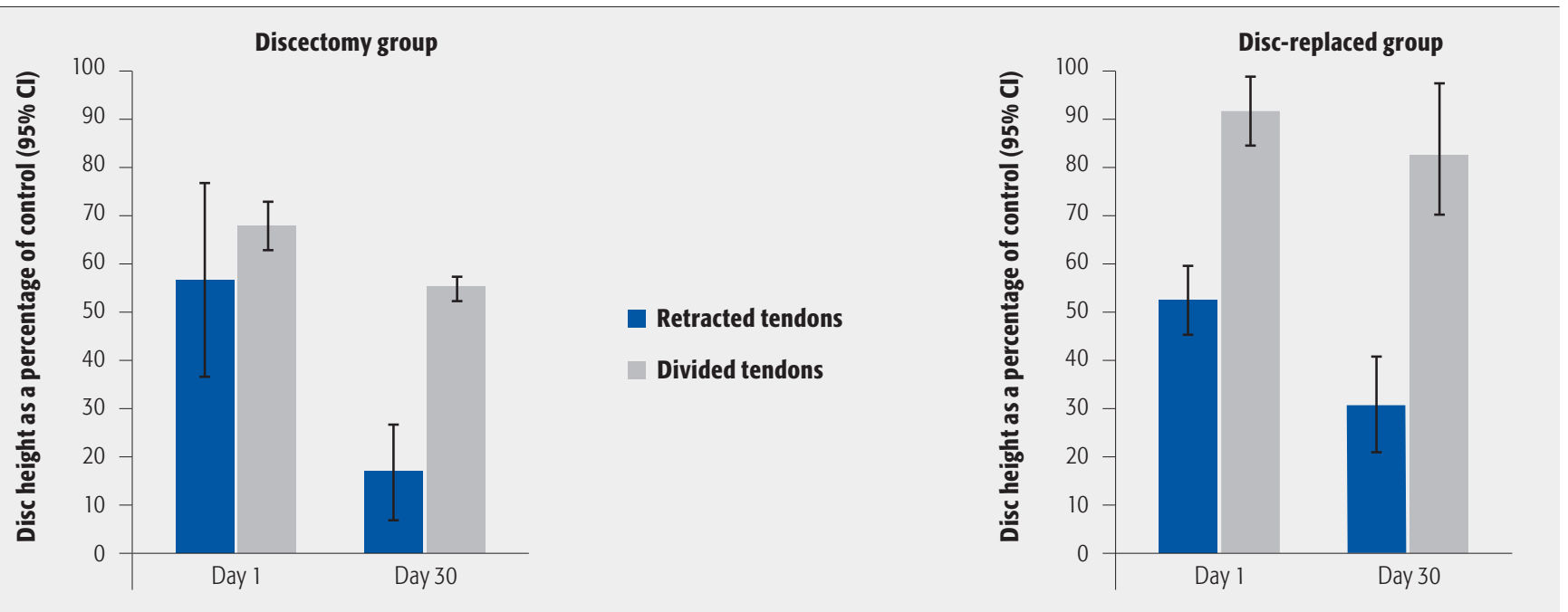


Table 1 Spontaneous tail movement within 30-minute periods of day and night.*

\begin{tabular}{|c|c|c|c|c|c|c|c|}
\hline \multirow{2}{*}{$\begin{array}{l}\text { Direction of } \\
\text { movement }\end{array}$} & \multirow{2}{*}{$\begin{array}{l}\text { Nonoperated } \\
\text { control group }\end{array}$} & \multicolumn{3}{|c|}{ Discectomy } & \multicolumn{3}{|c|}{ Reinsertion } \\
\hline & & Retracted group & Divided group & $\boldsymbol{P}^{*}$ & Retracted group & Divided group & $\boldsymbol{P}^{*}$ \\
\hline Vertical & $0.8( \pm 0.8)$ & $1.3( \pm 1.0)$ & $1.6( \pm 1.0)$ & .32 & $1.4( \pm 1.1)$ & $1.3(1.0 \pm 1.0)$ & .79 \\
\hline Left & $18.5( \pm 1.2)$ & $28.8( \pm 3.7)$ & $24.9( \pm 7.2)$ & .79 & $21.5( \pm .9)$ & $21.9( \pm 2.9)$ & .88 \\
\hline Right & $13( \pm 2.8)$ & $16.2( \pm 2.5)$ & $16.7( \pm 2.6)$ & .87 & $14.6( \pm .7)$ & $16.5( \pm 4.3)$ & .51 \\
\hline
\end{tabular}

*Values are mean (standard error).

Table 2 Semiquantitative scoring system of biological intervertebral disc characteristics.

\begin{tabular}{clc}
\hline Disc height as a percentage of control & T2 signal $^{*}$ & Semiquantitative grade \\
\hline \multirow{2}{*}{$75-100$} & $(+++)$ & 4 \\
\cline { 2 - 3 } & $(-)$ or $(+)$ or $(++)$ & 3 \\
\hline $25-74$ & Any & 1 \\
\hline $0-24$ & $(+)$ or $(++)$ or $(+++)$ & 0 \\
\cline { 2 - 2 } & $(-)$ & \\
\hline
\end{tabular}

${ }^{*}$ When T2 signal is absent (-); poor (+); fair (++); or strong (+++). See Fig 6.

Fig 10 Plot of semiquantitative scores.

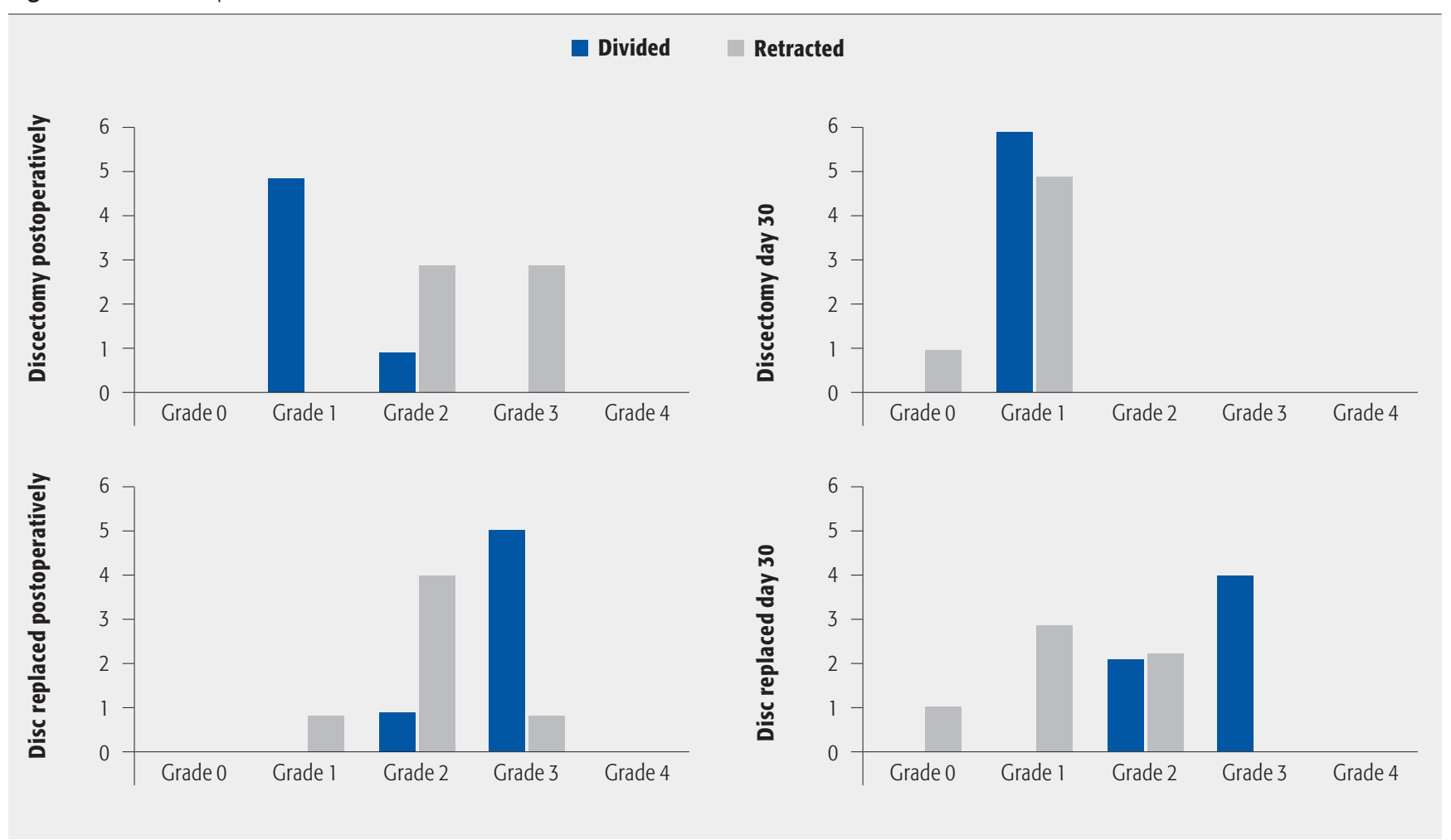




\section{RESULTS}

- Clinical

- There were no surgical-site complications (despite immunocompromised animals).

- No vascular injuries (capillary return, maintained vessel structure).

- Spontaneous tail movements were predominantly lateral, and the total number of tail movements was not significantly affected by surgical technique (Table 1).

- 7T MRI high-resolution imaging allowed disc assessment.

- Technique 1 led to DSH collapse even when the native IVD was replaced. While in Technique 2 , native IVD reinsertion significantly maintained disc height (Fig 9).

- Assessment of disc hydration led to development of a semiquantitative scoring system (Table $\mathbf{2}$ and Fig 6). Results (Fig 10) showed that it was possible to differentiate between the MRI appearances.

- Following discectomy (either technique) resulted in Grade 0 or 1 on day 30.

- Disc replacement with Technique 2 (divided) scored higher postoperatively and maintained this through day 30 with a Grade 2 or 3.

- Technique 1: Even with disc replacement (retracted), the grading deteriorated mainly to Grades 0 and 1 on day 30.

- No reimplanted disc had been displaced at day 30. - MRI revealed no vascular injuries.

- Micro CT: the surgical technique avoided mechanical damage to the vertebral end plates and bodies (Fig 8).

\section{DISCUSSION}

- Subcutaneous implantation of tissue-engineered IVD has led to implant survival with matrix production and propagated as a next step, the idea of an orthotopic model [3] for biological IVD replacement.

- The rat tail approach is reproducible and without marked complications. Surgery is less invasive, with similar outcomes, compared to preliminary data from other strategies in the rat lumbar spine [6].

- The tendon-incising technique has allowed good restoration of normal anatomy and maintains function, while tendon retraction leads to a nonrestorable collapse.

- We acknowledge that small animal models are a preliminary step and there are hurdles to translate the results in other species.

- Although it is not a bipedal model, orthotopic positioning allows assessment of intervertbral segment motion and thus demonstrates the effect of some mechanical loading on discs as shown in previous studies [7-9].

- A weakness of this feasibility study is the presence of notochordal cells in rat tail IVDs themselves [8]. However, the purpose of this model is to replace the implants in future research by various tissue-engineered constructs; so the texture of native IVDs becomes irrelevant.

- 7T MRI studies assess clinically relevant characteristics of the IVD. Structural changes are observable in the discs which are less with Technique 2 (divided).

\section{SUMMARY AND CONLCUSIONS}

- The athymic rat tail model, with a tendon-dividing surgical technique, can function as an orthotopic animal model for IVD research.

- Mechanical stimulation is maintained by preserved tail movements.

- 7T MRI is a feasible modality for longitudinal monitoring of the rat caudal disc.

\section{ACKNOWLEDGMENTS}

We thank AOSpine for their support to Harry Gebhard and Andrew R James. We also thank Matthew E Cunningham, A John Tsiouris, and Eric Aronowitz for their assistance in this study. Drawing created by Matthew Holt. 


\section{REFERENCES}

1. Bowles $\mathbf{R}$, Williams $\mathbf{R}$, Zipfel $\mathbf{W}$, et al (2010) Self-assembly of aligned tissue-engineered annulus fibrosus and intervertebral disc composite via collagen gel contraction. Tissue Eng Part A; 16:1339-1348.

2. Nesti LJ, Li WJ, Shanti RM, et al (2008) Intervertebral disc tissue engineering using a novel hyaluronic acid-nanofibrous scaffold (HANFS) amalgam. Tissue Eng Part A; 14(9):1527-1537.

3. Mizuno H, Roy AK, Zaporojan V, et al (2006) Biomechanical and biochemical characterization of composite tissue-engineered intervertebral discs. Biomaterials; 27(3):362-370.

4. Nerurkar NL, Sen S, Huang AH, et al (2010) Engineered disc-like angle-ply structures for intervertebral disc replacement. Spine (Phila Pa 1976); 35:867-873.

5. Zhang H, La Marca F, Hollister S, et al (2009) Developing consistently reproducible intervertebral disc degeneration at rat caudal spine by using needle puncture. J Neurosurg Spine; 10:522-530.

6. Bowles R, Gebhard H, Dyke J, et al (2010) Transplantation of Composite Tissue Engineered Intervertebral Discs to restore Function to the Rat Spine. In ORS: New Orleans, LA.

7. Oshima H, Ishihara H, Urban JP, et al (1993) The use of coccygeal discs to study intervertebral disc metabolism. J Orthop Res; 11(3):332338.

8. Sarver JJ, Elliott DM (2005) Mechanical differences between lumbar and tail discs in the mouse. J Orthop Res; 23(1):150-155.

9. MacLean JJ, Lee CR, Alini M, et al (2005) The effects of short-term load duration on anabolic and catabolic gene expression in the rat tail intervertebral disc. J Orthop Res; 23(5):11201127.

\section{EDITORIAL STAFF PERSPECTIVE}

The athymic rat tail model does appear to provide a potential animal model for future investigation into intervertebral disc replacement with appropriate technique in this prospective randomized animal study. This paper clearly demonstrates sharp dissection is superior to blunt detachment to maintaining intervertebral disc height, an interesting tangent as we learn more about soft-tissue dissection and maintenance of viable margins. There are, however, some significant limitations to this study.

One limitation lies in the inability of the rat tail model to provide significant axial loading upon the discs as would be the case in bipedal animals or in the actual spinal column of the investigated animals, as mentioned by the authors. In addition, the short-time frame of follow-up is of concern. Longer outcomes involving athymic rats may be necessary in order to understand if the replanted discs can withstand longer durations than just the 30 days. A 90-day window or even longer would seem to be more relevant to establish ingrowth and cartilage cell survival.

Another area of concern is the lack of significant data and limited discussion on the movements of the rats' tails following discectomy or reinsertion. None of the data in Table 1 demonstrated significance $(P<.05)$ from the control to the operated rats in regard to movement.

Detailed analysis of the results of the disc replacement or removal with clinical assessments, function, and imaging with MRI and micro CT is clearly one of the strengths of this paper. The comprehensive approach to imaging allows for very clear comparisons between the two surgical approaches of discs that were replanted or discarded.

There is no question of the importance of developing an appropriate animal model for the future quest for biological intervertebral disc replacements in an in vivo setting. A workable animal model will certainly facilitate future research opportunities and development of the disc replacements. Whether the athymic rat tail model will turn out to be the optimal model or not, this novel model certainly presents a laudable launching point for further investigations. 